\title{
Percepção de estudantes de Odontologia sobre recessão gengival
}

\author{
Dental student's perception about gingival recession \\ Percepción de los estudiantes de Odontología en la recesión gingival \\ Waleska Ohana de SOUZA-MELO' \\ ${ }^{I}$ Mestranda; Programa de Pós-Graduação em Odontologia, Universidade Estadual da Paraíba (UEPB), \\ 58429-500 Campina Grande-PB, Brasil \\ ${ }^{2}$ Graduada em Odontologia, Departamento de Odontologia da Universidade Estadual da Paraíba, \\ 58429-500 Campina Grande-PB, Brasil \\ ${ }^{3}$ Professora Doutora de Estomatologia; Departamento de Odontologia da Universidade Estadual da Paraíba, \\ 58429-500 Campina Grande-PB, Brasil \\ ${ }^{4}$ Professora Doutora de Periodontia; Departamento de Odontologia da Universidade Estadual da Paraíba, \\ 58429-500 Campina Grande-PB, Brasil
}

\section{Resumo}

Objetivo: Avaliar a percepção quanto às recessões gengivais de estudantes de Odontologia de uma Universidade da Paraíba. Material e Método: Tratou-se de uma pesquisa transversal com análise descritiva e analítica, tendo como instrumento um questionário estruturado sobre a etiologia e classificação das recessões gengivais, bem como a indicação de procedimentos terapêuticos das mesmas. A amostra foi obtida por conveniência, composta pelos estudantes regularmente matriculados do sétimo ao décimo períodos, destes, um total de 94 de 110 respondeu ao questionário. Resultados: A maioria dos estudantes (54,26\%) não tinha conhecimento sobre a classificação de recessão gengival de Miller. Entretanto, grande parte deles obteve êxito ao afirmar que o tabagismo, a higiene bucal e o tratamento ortodôntico têm influência sobre as recessões gengivais. Além disso, no que diz respeito à escolha do tratamento, 93,6\% dos estudantes afirmaram haver relação dependente com a etiologia da recessão. A partir da avaliação das respostas, observou-se que os estudantes do $7^{\circ}$ período apresentaram a maior média de acertos, seguidos dos $8^{\circ}, 10^{\circ}$ e $9^{\circ}$ períodos, no entanto, não houve diferença estatisticamente significativa entre os períodos. A análise dos resultados mostrou ainda que não há consenso entre os estudantes a respeito de quais condutas seriam recomendadas para o tratamento e prevenção das recessões gengivais. Conclusão: Dentro dos limites do presente estudo, pôde-se concluir que há necessidade de um melhor entendimento dos mesmos, sugerindo-se uma melhor abordagem teórico-prática referente ao assunto.

Descritores: Conhecimentos, Atitudes e Prática em Saúde; Educação em Odontologia; Estudantes de Odontologia; Periodontia; Retração Gengival.

\section{Abstract}

Objective: Evaluate the perception about gingival recessions of Dentistry students from a university of Paraíba. Material and Method: It was a cross-sectional research with descriptive and analytical analysis, with a structured questionnaire about the etiology and classification of gingival recessions, as well as the indication of therapeutic procedures for them. The sample was obtained for convenience, composed of students regularly enrolled in seventh to tenth periods, of whom 94 out of 110 answered the questionnaire. Results: The majority of students $(54.26 \%)$ had no knowledge about Miller's ginival recession classification. However, most of them were sucessful in affirming that smoking, oral hygiene and orthodontic treatment have na influence on gingival recessions. In addition, regarding the choice of treatment, $93,6 \%$ of the students affirm that there was a relationship dependet on the etiology of the recessions. From the evaluation of the answers, it was observed that students of the 7th period presented the highest average of hits, followed by the 8th, 10th and 9th periods, however, there was no statistically significant difference between the periods. The analysis of the results also showed that there is no consensus among the students concerning which behaviors would be recommended for the treatment and prevention of gingival recessions. Conclusion: Within the limits of this presente study, it was concluded that there is a need for a better understanding of them, suggesting a better theoretical-practical approach regarding the subject.

Descriptors: Health knowledge, Attitudes, Practice; Education, Dental; Students, Dental; Periodontics; Gingival Recession.

\section{Resumen}

Objetivo: Evaluar la percepción a cerca de las recesiones gingivales de estudiantes de Odontologia de una Universidad de Paraíba. Material y Método: Se trató de una búsqueda transversa con análisis descriptivo y analítico, teniendo como instrumento un cuestionario estructurado sobre la etiología y clasificación de las recesiones gingivales, así como la indicación de procedimientos terapéuticos de las mismas. La muestra fue obtenida por conveniencia, compuesta por los estudiantes regularmente matriculados del séptimo a lo décimo períodos, de éstos, un total de 94 de 110, respondieron al cuestionario. Resultados: La mayoría de los estudiantes (54.26\%) no tenían conocimiento de la clasificación de recesión gingival de Miller. Sin embargo, gran parte de ellos obtuvo éxito al afirmar que el tabaquismo, la higiene bucal y el tratamiento de ortodoncia tienen influencia sobre las recesiones gingivales. Además, en lo que refiere a la elección del tratamiento, 93.6\% de los estudiantes afirmaron haber relación dependiente con la etiología de la recesión. A partir de la evaluación de las respuestas, se observó que los estudiantes del $7^{\circ}$ período presentaron la mayor media de aciertos seguidos de $\operatorname{los} 8^{\circ}, 10^{\circ}$ y $9^{\circ}$ períodos, sin embargo, no hubo diferencia estadísticamente significativa entre los períodos. El análisis de los resultados, todavía mostro que no hay consenso entre los estudiantes acerca de qué conductas serian recomendadas para el tratamiento y prevención de las recesiones gingivales. Conclusión: Dentro de los límites del presente estudio, se pudio concluir que hay necesidad de un mejor entendimiento de los mismos, se sugiere un mejor abordaje teórica y práctico referente al asunto.

Descriptores: Conocimientos, Actitudes y Práctica en Salud; Educación en Odontología; Estudiantes de Odontología; Periodoncia; Recesión Gingival.

\section{INTRODUÇÃO}

Com a mudança de paradigmas na prática odontológica, a odontologia estética tem evoluído para uma abordagem interdisciplinar de vários tratamentos para atender às expectativas dos pacientes ${ }^{1}$. É cada vez maior a quantidade de novas informações referentes ao tratamento estético dental, estendendo-se para além da substituição e alteração da cor e forma dos dentes, passando a incluir o componente de tecido mole. Uma das preocupações estéticas mais comuns, associada com tecidos periodontais, é a recessão gengival ${ }^{2}$.
A recessão gengival é a exposição das raízes devido à migração apical das margens do tecido gengival, com exposição clínica da junção amelocementária ${ }^{3}$, podendo estar presente em ambos os arcos, nas faces vestibular e lingual e em qualquer dente ${ }^{4}$, favorecendo aos pacientes com essa condição o aumento do comprimento dos dentes ${ }^{5}$.

Por ser um defeito mucogengival, considerado um fenômeno interessante e complexo ${ }^{6}$, as recessões gengivais podem provocar: um aspecto antiestético, hipersensibilidade 
radicular, impactação alimentar, predisposição a cáries radiculares, dor durante a mastigação ou escovação e dificuldade no controle de biofilme, fatores esses que podem colaborar na progressão da doença periodontal ${ }^{7}$. É importante detectar a presença e o tipo de recessão gengival, e em seguida, diagnosticar a origem ou causa, a fim de removê-la, e assim tratá-la eficazmente . $^{8}$

Alguns tratamentos têm sido propostos e muitas técnicas cirúrgicas foram desenvolvidas, mas ainda não há consenso sobre o protocolo clínico para o tratamento de recessão gengival ${ }^{9}$. As terapias mais comuns para essas lesões referem-se à prevenção dos fatores etiológicos, ajustes oclusais, reorientações na escovação e procedimentos restauradores, além das técnicas cirúrgicas para recobrimento radicular $^{10}$

Considerando a importância da ampliação do conhecimento dos estudantes de Odontologia, bem como o levantamento das informações acerca das práticas dos mesmos frente aos desafios clínicos nos casos de recessões gengivais, o objetivo deste estudo foi avaliar a percepção desses estudantes sobre recessões gengivais, levando em consideração etiologia, classificação, bem como o tipo de conduta utilizado.

\section{MATERIAL E MÉTODO}

Tratou-se de um estudo transversal descritivo. A amostragem desse estudo foi obtida por conveniência, composta pelos alunos regularmente matriculados nos $7^{\circ}, 8^{\circ}$, $9^{\circ}$ e $10^{\circ}$ períodos do curso de Odontologia de uma Universidade da Paraíba. A amostra foi constituída por 94 estudantes de um universo de 110.

$\mathrm{O}$ estudo foi fundamentado em uma coleta de dados por meio de um questionário estruturado, que envolveu informações pertinentes à recessão gengival, tais como a sua etiologia e a conduta terapêutica, a partir de 17 questões objetivas, com algumas delas concedendo a possibilidade de múltiplas escolhas de resposta.

Para a elaboração do instrumento de coleta de dados do presente estudo, foram utilizados como referências os questionários Grover et al. ${ }^{2}$ e Casusa et al. ${ }^{11}$.

Para o aprimoramento do questionário, foi realizado um estudo piloto com aproximadamente $15 \%$ da amostra a ser estudada, constituída por 16 estudantes matriculados do sétimo ao décimo período (quatro de cada período).

Para a análise dos resultados, inicialmente, foi realizada a estatística descritiva e verificou-se a distribuição dos estudantes de cada período de acordo com as respostas assinaladas no questionário. Apenas as respostas corretas foram discriminadas nas tabelas, sendo que as demais respostas foram agrupadas na categoria "outras". Definido o critério de correção para as questões, o número de acertos foi somado para cada estudante, sendo que esses valores poderiam oscilar ou variar entre um e 16. Por fim, empregouse o teste ANOVA para comparar o número de acerto dos estudantes de cada período. O nível de significância foi fixado em $\mathrm{p}<0,05$. Todas as análises foram realizadas usando o software Statistical Package for Social Sciences (SPSS for Windows), versão 20.0 (IBM Inc, Amonk, NY, USA) e considerando um intervalo de confiança de $95 \%$.

Em respeito à Resolução 466/12 do Conselho Nacional de Saúde $(\mathrm{CNS} / \mathrm{MS})^{12}$, que regulamenta a ética na pesquisa envolvendo seres humanos no Brasil, este estudo foi submetido à análise do Comitê de Ética em Pesquisa (CEP) com Seres Humanos da Universidade Estadual da Paraíba (UEPB) e aprovado pelo parecer CAEE 45729715.9.0000. 5187. Os participantes do estudo foram informados quanto ao tipo de questionário no qual seriam submetidos, e tiveram sua participação aceita mediante a assinatura do Termo de Consentimento Livre e Esclarecido (TCLE).

\section{RESULTADOS}

Participaram do estudo 94 estudantes do curso de Odontologia matriculados no $7^{\circ}(\mathrm{n}=25 ; 26,6 \%), 8^{\circ}(\mathrm{n}=22$; $23,4 \%), 9^{\circ}(\mathrm{n}=29 ; 30,9 \%)$ e $10^{\circ}(\mathrm{n}=18 ; 19,1 \%)$ períodos. Destes, 45,7\% ( $\mathrm{n}=43)$ afirmaram conhecer a classificação de Miller (1985) para as recessões gengivais (Gráfico 1).

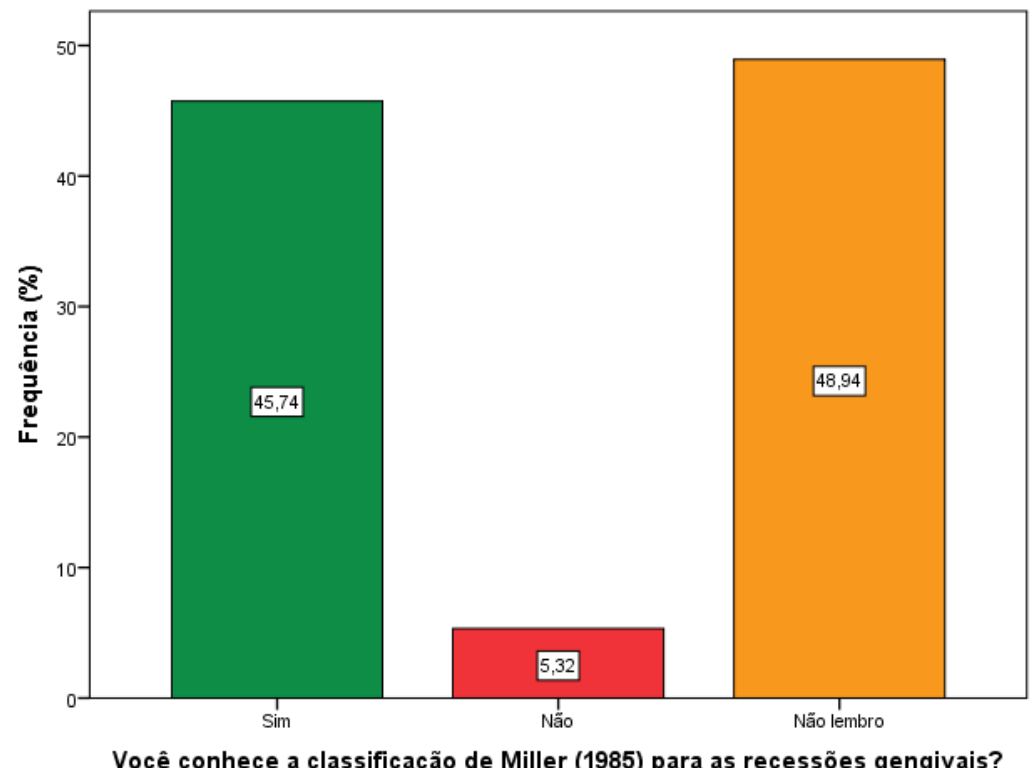

Gráfico 1: Distribuição percentual dos estudantes de acordo com a resposta assinalada na pergunta sobre conhecer a classificação de Miller para recessões gengivais

A Tabela 1 exibe a distribuição dos estudantes de cada período de acordo com as respostas assinaladas no questionário. A maioria deles assinalou que a classificação I de recessão gengival de Miller tem um melhor prognóstico (n $=75 ; 79,8 \%)$, bem como que a higiene oral $(\mathrm{n}=92 ; 97,9 \%) \mathrm{e}$ o tabagismo $(n=83 ; 88,3 \%)$ influenciam a progressão da recessão gengival. Questionados sobre qual a conduta terapêutica frente a um paciente com uma recessão gengival no dente 45, Classe I, acrescido de sintomatologia dolorosa, apenas $3(16,7 \%)$ estudantes do $10^{\circ}$ período assinalaram o recobrimento radicular com enxerto livre com mucosa do palato.

A partir do Gráfico 2 é possível visualizar a distribuição da pontuação média dos estudantes de acordo com cada período. Após avaliar todas as respostas e somar a pontuação de cada estudante, observou-se que a média $( \pm$ desvio padrão) das notas dos alunos do $7^{\circ}, 8^{\circ}, 9^{\circ}$ e $10^{\circ}$ períodos, foram respectivamente: $8,38( \pm 2,10), 8,29( \pm 1,23)$, $7,83( \pm 2,08)$ e $7,85( \pm 1,83)$. Portanto, os estudantes do $7^{\circ}$ período apresentaram a maior média de acertos, seguidos pelos estudantes do $8^{\circ}, 10^{\circ}$ e $9^{\circ}$ períodos. No entanto, o teste ANOVA revelou que *não houve diferença estatisticamente significativa ao nível de $p<0,05(p=0,638)$.

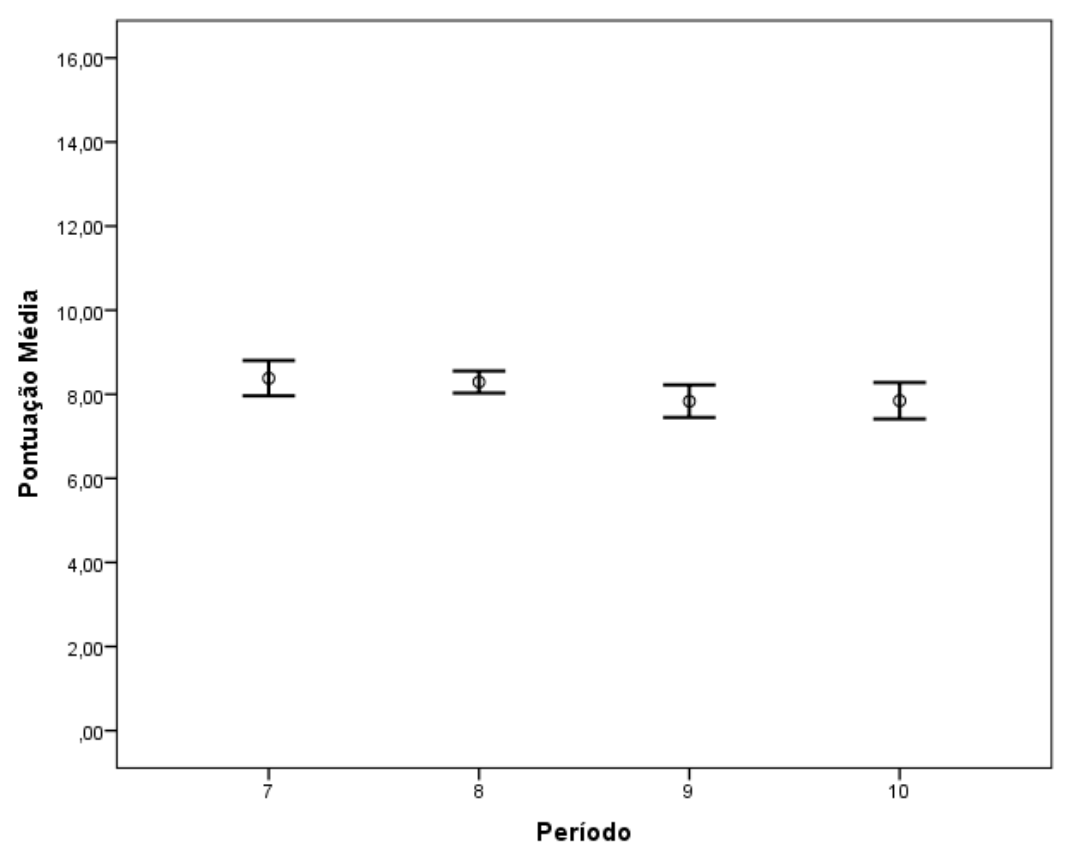

Gráfico 2. Distribuição da pontuação média dos alunos de acordo com cada período ( $p=0,638$, obtido por meio do teste ANOVA). 
Tabela 1. Distribuição dos estudantes de cada período de acordo com as respostas assinaladas no questionário

\begin{tabular}{|c|c|c|c|c|}
\hline \multirow[b]{3}{*}{ Variáveis } & \multicolumn{4}{|c|}{ Período } \\
\hline & 7 & 8 & 9 & 10 \\
\hline & $n(\%)$ & $\mathrm{n}(\%)$ & $n(\%)$ & $\mathrm{n}(\%)$ \\
\hline \multicolumn{5}{|c|}{$\begin{array}{l}\text { 2. Qual o tipo de recessão de Miller tem um melhor } \\
\text { prognóstico? }\end{array}$} \\
\hline Classificação I & $18(72,0)$ & $20(90,9)$ & $\begin{array}{c}24 \\
(82,8)\end{array}$ & $13(72,2)$ \\
\hline Outras respostas & $7(28,0)$ & $2(9,1)$ & $5(17,2)$ & $5(27,8)$ \\
\hline $\begin{array}{l}\text { 3. Ainda referente a questão anterior, qu } \\
\text { Maior quantidade de gengiva nas } \\
\text { laterais/ A recessão não atingiu a linha } \\
\text { muco gengival / Existe preservação das } \\
\text { papilas/ Presença de osso e, } \\
\text { consequentemente, } \\
\text { subjacente } \\
\text { Outras respostas }\end{array}$ & $\begin{array}{l}\text { al o motivo } \\
8(32,0)\end{array}$ & $\begin{array}{l}\text { ue o (a) lev } \\
1(4,5)\end{array}$ & $\begin{array}{l}\text { a pensar a } \\
2(6,9)\end{array}$ & $3(16,7)$ \\
\hline \multicolumn{5}{|c|}{ 4. A higiene oral influencia a progressão das recessões gengivais? $(30,1)$} \\
\hline Sim & $24(96,0)$ & $22(100,0)$ & $\begin{array}{c}28 \\
(96.6)\end{array}$ & $\begin{array}{c}18 \\
(100,0)\end{array}$ \\
\hline Não & $1(4,0)$ & $0(0,0)$ & $1(3,4)$ & $0(0,0)$ \\
\hline \multicolumn{5}{|c|}{$\begin{array}{l}\text { 5. Existe associação do tipo de cerdas da escova dental (macia, média, dura) e/ou } \\
\text { potência/força de escovação com a recessão gengival? }\end{array}$} \\
\hline $\begin{array}{l}\text { Depende do biótipo periodontal do } \\
\text { paciente }\end{array}$ & $8(32,0)$ & $5(22,7)$ & $7(24,1)$ & $4(22,2)$ \\
\hline Outras respostas & $17(68,0)$ & $17(77,3)$ & $\begin{array}{c}22 \\
(75,9)\end{array}$ & $14(77,8)$ \\
\hline \multicolumn{5}{|c|}{ 6. 0 tabagismo influencia a progressão das recessões gengivais? } \\
\hline $\operatorname{sim}$ & $21(84,0)$ & $21(95,5)$ & $\begin{array}{c}24 \\
(82,8)\end{array}$ & $17(94,4)$ \\
\hline Não & $4(16,0)$ & $1(4,5)$ & $5(17,2)$ & $1(5,6)$ \\
\hline \multicolumn{5}{|c|}{ 7. O tratamento ortodôntico causa recessão gengival? } \\
\hline Em alguns casos & $24(96,0)$ & $21(95,5)$ & $\begin{array}{c}25 \\
(86,2)\end{array}$ & $\begin{array}{c}18 \\
(100,0)\end{array}$ \\
\hline Outras respostas & $1(4,0)$ & $1(4,5)$ & $4(13,8)$ & $0(0,0)$ \\
\hline \multicolumn{5}{|c|}{ 8. Qual a causa mais comum para recessão gengival? } \\
\hline $\begin{array}{l}\text { Trauma mecânico (técnica de higiene } \\
\text { oral inadequada) }\end{array}$ & $4(16,0)$ & $8(36,4)$ & $\begin{array}{c}13 \\
(44,8)\end{array}$ & $8(44,4)$ \\
\hline Outras respostas & $21(84,0)$ & $14(63,6)$ & $\begin{array}{c}16 \\
(55,2)\end{array}$ & $10(55,6)$ \\
\hline \multicolumn{5}{|c|}{ 9. O que se deve fazer para prevenir as recessões gengivais? } \\
\hline $\begin{array}{l}\text { Consultas periódicas ao dentista para } \\
\text { controle clíico das áreas de risco já } \\
\text { detectadas }\end{array}$ & $19(76,0)$ & $14(63,6)$ & $\begin{array}{c}14 \\
(48,3)\end{array}$ & $8(44,4)$ \\
\hline Outras respostas & $6(24,0)$ & $8(36,4)$ & & $10(55,6)$ \\
\hline \multicolumn{5}{|c|}{$\begin{array}{l}\text { 10. Qual conduta clínica poderia ser utilizada de forma preventiva e/ou conservadora, } \\
\text { nos casos de recessão gengival? } \\
\text { Raspagem }\end{array}$} \\
\hline $\begin{array}{l}\text { Raspagem e } \quad \text { alisamento } \\
\text { coronoradicular/ Avaliação sistemática } \\
\text { das inserçōes musculares e freios/ } \\
\text { Cirurgia de freio e inserçōes musculares } \\
\text { hipertrófico }\end{array}$ & $0(0,0)$ & $1(4,5)$ & $0(0,0)$ & $2(11,1)$ \\
\hline Outras respostas & $\begin{array}{c}25 \\
(100,0)\end{array}$ & $21(95,5)$ & $\begin{array}{c}29 \\
(100,0)\end{array}$ & $16(88,9)$ \\
\hline \multicolumn{5}{|c|}{$\begin{array}{l}\text { 11. Das alternativas abaixo, qual a sua principal indicação para a realização de um } \\
\text { recobrimento radicular em virtude de uma recessão gengival? }\end{array}$} \\
\hline $\begin{array}{l}\text { Estética associada à sensibilidade } \\
\text { dentinária }\end{array}$ & $12(48,0)$ & $13(59,1)$ & $\begin{array}{c}17 \\
(58,6)\end{array}$ & $6(33,3)$ \\
\hline Outras respostas & $13(52,0)$ & $9(40,9)$ & $\begin{array}{c}12 \\
(41,4)\end{array}$ & $12(66,7)$ \\
\hline \multicolumn{5}{|c|}{$n$ tratamento para recessõe } \\
\hline $\begin{array}{l}\text { Em qualquer idade, dependendo da } \\
\text { queixa do paciente }\end{array}$ & $17(68,0)$ & $16(72,7)$ & & $14(77,8)$ \\
\hline Outras respostas & $8(32,0)$ & $6(27,3)$ & $8(27,6)$ & $4(22,2)$ \\
\hline \multicolumn{5}{|c|}{$\begin{array}{l}\text { 13. A etiologia das recessões gengivais influencia na tomada de decisão quanto à } \\
\text { terapia a ser empregada em uma recessão gengival? }\end{array}$} \\
\hline $\operatorname{sim}$ & $22(88,0)$ & $22(100,0)$ & & $16(88,9)$ \\
\hline Não & $3(12,0)$ & $0(0,0)$ & $1(3,4)$ & $2(11,1)$ \\
\hline \multicolumn{5}{|c|}{$\begin{array}{l}\text { 14. Qual a sua conduta terapêutica frente a um paciente com uma recessão gengival no } \\
\text { dente 22, Classe I de Miller e sem sintomatologia dolorosa e presença de mucosa } \\
\text { inserida? }\end{array}$} \\
\hline $\begin{array}{l}\text { Recobrimento radicular a partir de } \\
\text { retalhos pediculados }\end{array}$ & $4(16,0)$ & $1(4,5)$ & $2(6,9)$ & $1(5,6)$ \\
\hline Outras respostas & $21(84,0)$ & $21(95,5)$ & $\begin{array}{c}27 \\
(93,1)\end{array}$ & $17(94,4)$ \\
\hline
\end{tabular}

15. Qual a sua conduta terapêutica frente a um paciente com uma recessão gengival no dente 22, Classe I, acrescido de sintomatologia dolorosa e presença de gengiva

inserida?
Recobrimento radicular a partir de
retalhos pediculados

$\begin{array}{lllll}\text { Outras respostas } & 18(72,0) & 20(90,9) & (82,8) & 15(83,3)\end{array}$

16. Qual a sua conduta terapêutica frente a um paciente com uma recessão gengival no dente 45 , Classe I, sem sintomatologia dolorosa?

$\begin{array}{lllll}\text { Recobrimento radicular com enxerto } & 4(16,0) & 0(0,0) & 3(10,3) & 0(0,0)\end{array}$

livre com mucosa do palato
Outras respostas

17. Qual a sua conduta terapêutica frente a um paciente com uma recessão gengival no dente 45 , Classe I, acrescido de sintomatologia dolorosa?

$\begin{array}{llllll}\begin{array}{l}\text { Recobrimento radicular com enxerto } \\ \text { livre com mucosa do palato }\end{array} & 10(40,0) & 1(4,5) & 2(6,9) & 3(16,7)\end{array}$

Outras respostas

$15(60,0) \quad 21(95,5) \quad(93,1)$

\section{DISCUSSÃO}

Ao longo dos últimos anos a Odontologia evoluiu de tal modo que os clínicos não são apenas necessários para tratar a doença e melhorar a função, mas também para lidar com as crescentes exigências estéticas dos seus pacientes ${ }^{13}$. A criação de uma boa estética exige uma análise dos pacientes na sua totalidade. A estética do paciente tem de ser contextualizada dentro da harmonia de todo o rosto, e o impacto visual do sorriso não pode ser associado exclusivamente à beleza de um ou mais dentes ${ }^{14}$. Dessa forma, os estudantes devem ser preparados para atender as expectativas e necessidades dos seus pacientes, tornando-se pesquisas sobre sua percepção de extrema importância.

Observou-se que o conhecimento da maioria dos estudantes, neste estudo, não condiz, em mais da metade das assertivas, com as atuais evidências científicas relacionadas à recessão gengival.

Entre os estudantes componentes da amostra, a maioria não tinha conhecimento sobre a classificação de recessão gengival de Miller. Outras classificações surgiram ao longo do tempo para categorizar esse defeito mucogengival, entretanto, a classificação de Miller constitui uma base importante na decisão da terapêutica a ser empregada em determinada situação, visto que ela é fundamentada na avaliação do prognóstico com relação à cobertura radicular ${ }^{2}$.

Em se tratando do melhor prognóstico, frente às classificações de Miller, muitos estudantes foram exitosos ao responder Classe I, corroborando com um estudo ${ }^{15}$ que concluiu em ensaio clínico um maior sucesso no recobrimento radicular completo em $85 \%$ dos casos de Classe I, 65\% Classe II e $16 \%$ Classe IV. Entretanto, no item subsequente, que abordava o motivo pelo qual a classificação assinalada teria o melhor prognóstico, observa-se uma interessante contradição, tendo apenas 14,9\% $(n=14)$ de acerto.

Em relação à progressão das recessões gengivais, foi questionada a influência de hábitos de higiene oral e tabagismo. Nestas assertivas a maioria dos estudantes respondeu positivamente em ambas. $\mathrm{O}$ controle de biofilme desempenha um papel essencial na prevenção da inflamação gengival, o que poderia desencadear ou acelerar a progressão da recessão ${ }^{16}$. Adicionalmente, Chrysanthakopoulos ${ }^{17}$ observou em seus achados uma associação entre tabagismo crônico e recessão gengival e mostrou um fator causal do tabagismo na doença periodontal. A ação do tabagismo como causador de recessão gengival pode ser, possivelmente, explicada através de alterações na resposta imune e mudanças locais, tais como diminuição da circulação gengival.

A prevalência dos acertos dos estudantes foi elevada quando afirmaram que o tratamento ortodôntico, em alguns casos, pode causar a recessão gengival, visto que apesar dos benefícios do tratamento ortodôntico, por vezes, durante o tratamento, pode se desenvolver uma recessão localizada, isto acontece quando a aplicação de forças excessivas impede o reparo e a capacidade de remodelação do osso alveolar ${ }^{3}$. Em um estudo caso controle com 588 pacientes, foi observada uma prevalência de $10,3 \%$ de recessões gengivais após o tratamento ortodôntico completo ${ }^{18}$.

Existem numerosas técnicas propostas para o tratamento de recessões únicas ou múltiplas ${ }^{19}$, quando questionados quanto a uma determinada conduta, a resposta mais indicada para melhor conduta clínica, como forma preventiva e/ou conservadora, nos casos de recessão gengival seria a junção de técnicas como avaliação das inserções musculares, raspagem e alisamento coronoradicular e cirurgia de freio hipertrófico, entretanto, apenas três estudantes optaram pelas condutas corretas, resultando em uma porcentagem muito baixa de acerto.

Ao serem questionados sobre o tratamento das recessões gengivais quanto à faixa etária, 68 estudantes $(63,92 \%)$ responderam que o mesmo deve ser feito em qualquer idade, dependendo da queixa do paciente, observando-se que a maioria respondeu de forma exitosa. Essa questão leva a uma reflexão, quanto a recessão gengival, levantada por Jati et al..$^{20}$, que durante décadas, acreditava-se que a recessão gengival era parte dos processos de envelhecimento humano, no entanto, todas as evidências que 
sustentam tal afirmação são bastante fracas, pois $o$ envelhecimento pode aumentar a possibilidade de as causas da retração gengival agir, mas isso não significa que elas sejam inerentes ao envelhecimento.

Após a avaliação das respostas e obtenção das médias, os resultados mostraram que os alunos do $7^{\circ}$ período obtiveram maior média $8,38( \pm 2,10)$, em relação aos demais períodos, isto pode ter ocorrido pelo fato de que, nesta Instituição, a disciplina de Periodontia é vista, isoladamente, nos $5^{\circ}$ e $6^{\circ}$ períodos, a partir do $7^{\circ}$ sendo implantada nas Clínicas Integradas, estando assim, os conteúdos mais recentes para tais alunos.

Diante disso, é notória a necessidade de se reforçar os estudos com os estudantes acerca desse tema, a fim de se observar quais as dúvidas dos mesmos em relação ao diagnóstico e ao tratamento, de modo que o paciente seja o maior beneficiado.

\section{CONCLUSÃO}

A análise dos resultados evidencia que não há consenso entre os estudantes no que diz respeito a conduta que seria recomendada para o tratamento e prevenção das recessões gengivais, bem como quanto ao conhecimento referente a etiologia e classificação das mesmas.

\section{REFERÊNCIAS}

1. Trivedi SR, Bhavsar NV, Dulani K, Trivedi R. Clinical evaluation of subepithelial connective tissue graft and guided tissue regeneration for treatment of Miller's class 1 gingival recession (comparative, split mouth, six months study). J Clin Exp Dent. 2014;6(3):e218-24.

2. Grover V, Kapoor A, Malhotra R, Sachdeva S. Interest and satisfaction of dentists in practicing periodontics: A survey based on treatment of gingival recession. Dent Res J (Isfahan). 2012; 9(4):404-13.

3. Rafiuddin S, Pradeep Kumar YG, Biswas S, Prabhu SS, Chandrashekar BM, Rakesh MP. Iatrogenic damage to the periodontium caused by orthodontic treatment procedures: an overview. Open Dent J. 2015; 9:228-34.

4. Yared KF, Zenobio EG, Pacheco W. Periodontal status of mandibular central incisors after orthodontic proclination in adults. Am J Orthod Dentofacial Orthop 2006; 130(1):6.e1-8.

5. Mufti S, Dadawala SM, Patel P, Shah M, Dave DH. Comparative evaluation of platelet-rich fibrin with connective tissue grafts in the treatment of miller's class i gingival recessions. Contemp Clin Dent. 2017; 8(4):531-7.

6. Kassab MM, Badawi H, Dentino AR. Treatment of gingival recession. Dent Clin North Am.2010; 54(1):129-40

7. Rodrigues DC, Alves R, Segundo TK. Emprego do enxerto de tecido conjuntivo subepitelial no recobrimento radicular. RGO, Rev Gaúch Odontol. 2010; 58(1):115-8.

8. Beltrán V, Silva M, Padilla M, Aillapan E, Sanhueza A, Cantín $M$ et al. Morphological Patterns of Gingival Recession in Adult Chilean Population. Int J Morphol. 2013; 31(4):1365-70.

9. Chambrone L, Sukekava F, Araújo MG, Pustiglioni FE, Chambrone LA, Lima LA. Root coverage procedures for the treatment of localised recession-type defects: a Cochrane systematic review. J Periodontol. 2010; 81(4):452-78

10. Guida B, Nasciben M, Carvalho E, Ribeiro EDP. Recobrimento radicular de recessões gengivais associados a lesões cervicais não cariosas - revisão da literatura. R Periodontia. 2010; 20(2):14-21.

11. Casusa AAL, Silva ELMS, Pinheiro RCQ, Ribeiro ILA, Lucena KCR. Percepção dos acadêmicos de odontologia quanto à conduta clínica frente às recessões gengivais. Perionews. 2015; 9(4):335-40.

12. Brasil, Ministério da Saúde. Resolução 466/2012 do Conselho Nacional de Saúde/MS Sobre Diretrizes e Normas Regulamentadoras de Pesquisa envolvendo seres humanos. Diário Oficial da União, 12 de dezembro de 2012.

13. Bhusari P, Agrawal N, Upadhyay S, Verma S, Jain A, Jaroli S. Classification \& prevalence of dental surface defects in areas of gingival recession- a clinical study. J Clin Diagn Res. 2014; 8(7):1-4.

14. Zuchelli G, Sharma P, Mounssif I. Esthetics in periodontics and implantology. Periodontology 2000. 2018; 77(1):7-18.

15. Vergara JA, Cafesse RG. Localized gingival recessions treated with the original envelope technique: a report of 50 consecutive patiens. J Periodontol. 2004; 75(10):1397-403

16. Andrade RN, Tôrres FR, Ferreira RF, Catharino F. Treatment of anterior crossbite and its influence on gingival recession. RGO, Rev Gaúch Odontol. 2014; 62(4):411-16.

17. Chrysanthakopoulos NA. Gingival Recession and Smoking in Young Adults: A Crosssectional Survey. J Periodontol Implant Dent. 2010; 2(2):77-82.

18. Vasconcelos G, Kjellsen K, Preus H, VandevskaRadunovic V, Hansen BF. Prevalence and severity of vestibular recession in mandibular incisors after orthodontic treatment: a case-control retrospective study. Angle Orthod. 2012; 82(1):42-7.

19. Morashini V, Barboza ESP. Use of platelet-rich fibrin membrane in the treatment of gingival recession: a systematic review and meta-analysis. J Periodontol. 2016; 87(3):281-90.

20. Jati AS, Furquim LZ, Consolaro A. Gingival recession: its causes and types, and the importance of orthodontic treatment. Dental Press J Orthod. 2016;21(3):18-29

\section{CONFLITO DE INTERESSES}

Os autores declaram não haver conflitos de interesse.

\section{AUTOR PARA CORRESPONDÊNCIA}

Waleska Ohana de Souza Melo

waleska.ohana@gmail.com

Submetido em 18/04/2018 Aceito em 11/05/2018 